\title{
AN UPPER BOUND OF THE MEAN GROWTH IN THE WILLIAMS-BJERKNES TUMOUR MODEL
}

\author{
F. MARTINEZ and A.R. VILLENA \\ Received December 1, 1998 and, in revised form, June 29, 1999
}

\begin{abstract}
We consider a cancer started with a single cancerous cell which spreads through an epithelial basal layer according to the WilliamsBjerknes tumour model on the lattice $\mathbb{Z}^{2}$. We prove that the expected number $\mu(t)$ of cancerous cells at time $t$ satisfies $\lim _{t \rightarrow+\infty} \frac{\mu(t)}{t^{\rho}}=0$ for all $\rho>2$.
\end{abstract}

\section{Introduction}

Based on chemical tests and mitotic patterns, Williams and Bjerknes proposed in [8] a model for the cancer spread through an epithelial basal layer (see also [5]). Independently, the Williams-Bjerknes tumour model was formulated within the field of interacting particle systems as the biased voter model (see [7]).

We consider the spread of cancerous cells started with a single cancerous cell at the origin through the basal layer of an epithelium modeled on

1991 Mathematics Subject Classification. 60K35, 92C50.

Key words and phrases. Williams-Bjerknes tumour model, expected number of cancerous cells.

ISSN 1425-6908 (c) Heldermann Verlag. 
the lattice $\mathbb{Z}^{2}$. The set $\xi_{t}^{A}$ of sites occupied by cancerous cells at time $t$, given that the initial state is $A$, is a Markov proces on the state space \{finite subsets of $\left.\mathbb{Z}^{2}\right\}$ thoroughly studied in [1-3], [6]. Bramson and Griffeath showed that the tumour has a linear rate of radial growth and this suggests that the expected number of cancerous cells has a quadratic rate of growth. In this paper we give a very basic proof, without involve the outstanding results of Bramson and Griffeath, of the following theorem on the asymptotic behaviour of the mean tumour growth.

Theorem. Let $\mu(t)$ be the expected number of cancerous cells at time $t$ for the Williams-Bjerknes tumour model starting from a single cancerous cell. Then

$$
\lim _{t \rightarrow+\infty} \frac{\mu(t)}{t^{\rho}}=0
$$

for all $\rho>2$.

\section{The tumour growth model}

Cells are assumed to be of two types, normal and cancerous, and are located on a suitable lattice, one at each site. With each celular division, one daughter remains in the site, while the other displaces a neighbouring cell which is pushed out of the basal layer. Cancerous cells are assumed to divide at a faster rate than normal cells. Splitting times for both normal and cancerous cells are assumed to be independent and have exponential distributions with parameter 1 and $\kappa>1$, respectively. This makes the probability that a normal cell will split in the time interval $[t, t+\Delta t]$ equals $\Delta t$, irrespective of the time since its last division. For the cancerous cells, this event occurs with probability $\kappa \Delta t$.

\section{Differential inequalities for the tumour model}

Cells are situated on the lattice $\mathbb{Z}^{2}$. For each $i \in \mathbb{Z}^{2}$ the neighbours of $i$ are given by

$$
\omega_{i}=\left\{\left(i_{1}-1, i_{2}\right),\left(i_{1}, i_{2}-1\right),\left(i_{1}+1, i_{2}\right),\left(i_{1}, i_{2}+1\right)\right\},
$$

$p_{i}(t)$ stands for the probability that the cell situated at $i$ is cancerous at time $t$ and $\mu(t)=\sum_{i \in \mathbb{Z}^{2}} p_{i}(t)$ is the expected number of cancerous cells at time $t$.

For the cell situated at $i$ to be cancerous at time $t+\Delta t$, either it is cancerous at time $t$ and no normal neighbouring cell displaces it in the time interval $[t, t+\Delta t]$, or else it is normal at time $t$ and a cancerous neighbouring 
cell splits and displaces it in $[t, t+\Delta t]$. Consequently, for each $i \in \mathbb{Z}^{2}$, the probability of the cell $i$ to be cancerous at time $t+\Delta t$ can be expressed as

$$
p_{i}(t+\Delta t)=p_{i}(t) u_{i}(t)+\left(1-p_{i}(t)\right)\left[\frac{\kappa \Delta t}{4} \sum_{j \in \omega_{i}} v_{j}(t)\right]+o(\Delta t),
$$

where we write $u_{i}(t)$ for the conditional probability that no normal neighbouring cell displaces the cell located at $i$ in the time interval $[t, t+\Delta t]$ given that the cell $i$ is cancerous and, for $j \in \omega_{i}$, we write $v_{j}(t)$ for the conditional probability that the cell located at $j$ is cancerous at time $t$ given that the cell located at $i$ is normal at time $t$. Since $u_{i}(t) \leq 1$, we have

$$
p_{i}(t+\Delta t) \leq p_{i}(t)+\left(1-p_{i}(t)\right)\left[\frac{\kappa \Delta t}{4} \sum_{j \in \omega_{i}} v_{j}(t)\right]+o(\Delta t) .
$$

On the other hand, for each $j \in \omega_{i}$, we have

$$
\left(1-p_{i}(t)\right) v_{j}(t)+p_{i}(t) w_{j}(t)=p_{j}(t),
$$

where $w_{j}(t)$ is the conditional probability that the cell located at $j$ is cancerous at time $t$ given that the cell located at $i$ is cancerous at time $t$. Thus $\left(1-p_{i}(t)\right) v_{j}(t) \leq p_{j}(t)$. Consequently,

$$
\frac{1}{4} \sum_{j \in \omega_{i}} v_{j}(t) \leq p_{i}(t) \frac{1}{4} \sum_{j \in \omega_{i}} v_{j}(t)+\frac{1}{4} \sum_{j \in \omega_{i}} p_{j}(t) .
$$

Since $\frac{1}{4} \sum_{j \in \omega_{i}} v_{j}(t) \leq 1$, we have

$$
\frac{1}{4} \sum_{j \in \omega_{i}} v_{j}(t) \leq p_{i}(t)+\frac{1}{4} \sum_{j \in \omega_{i}} p_{j}(t)
$$

and so

$$
\frac{p_{i}(t+\Delta t)-p_{i}(t)}{\Delta t} \leq\left(1-p_{i}(t)\right) \kappa\left[p_{i}(t)+\frac{1}{4} \sum_{j \in \omega_{i}} p_{j}(t)\right]+\frac{o(\Delta t)}{\Delta t} .
$$

Assume that the functions $p_{i}$ are differentiable for all $i \in \mathbb{Z}^{2}$ and let $\Delta t$ approach zero. This yields the following family of differential inequalities

$$
p_{i}^{\prime}(t) \leq \kappa\left(1-p_{i}(t)\right)\left[p_{i}(t)+\frac{1}{4} \sum_{j \in \omega_{i}} p_{j}(t)\right], \forall t \in\left[0,+\infty\left[, \forall i \in \mathbb{Z}^{2} .\right.\right.
$$

The initial state of the epithelium is given by $p_{0}(0)=1$ and $p_{i}(0)=0$ for all $i \in \mathbb{Z}^{2} \backslash\{0\}$. 
Lemma. $p_{i}(t) \leq 1-\exp \left(-2^{n} \kappa^{n} t^{n} / n !\right)$ for all $t \in\left[0,+\infty\left[, i \in \mathbb{Z}^{2} \backslash \Omega_{n-1}\right.\right.$, and $n \in \mathbb{N}$, where we write $\Omega_{n}$ for the set $\left\{j \in \mathbb{Z}^{2}:\left|j_{1}\right|+\left|j_{2}\right| \leq n\right\}$.

Proof. For all $t \in\left[0,+\infty\left[\right.\right.$ and $i \in \mathbb{Z}^{2}$, we have $\frac{1}{4} \sum_{j \in \omega_{i}} p_{j}(t) \leq 1$, and hence

$$
p_{i}^{\prime}(t) \leq 2 \kappa\left(1-p_{i}(t)\right)
$$

From this we deduce that

$$
p_{i}(t) \leq 2 \kappa t-2 \kappa \int_{0}^{t} p_{i}(s) d s,
$$

and Gronwall lemma $[4,10.5 .1 .3]$ gives $p_{i}(t) \leq 1-e^{-2 \kappa t}$ for all $t \in[0,+\infty[$ and $i \in \mathbb{Z}^{2} \backslash \Omega_{0}$.

Assume that $p_{i}(t) \leq 1-e^{-2^{n} \kappa^{n} t^{n} / n !}$ for all $t \in\left[0,+\infty\left[\right.\right.$ and $i \in \mathbb{Z}^{2} \backslash \Omega_{n-1}$. Let $i \in \mathbb{Z}^{2} \backslash \Omega_{n}$. Then $\omega_{i} \subset \mathbb{Z}^{2} \backslash \Omega_{n-1}$ and hence

$$
\begin{aligned}
p_{i}^{\prime}(t) & \leq \kappa\left(1-p_{i}(t)\right)\left[p_{i}(t)+\frac{1}{4} \sum_{j \in \omega_{i}} p_{j}(t)\right] \\
& \leq \kappa\left(1-p_{i}(t)\right)\left[\left(1-e^{-2^{n} \kappa^{n} t^{n} / n !}\right)+\frac{1}{4} \sum_{j \in \omega_{i}}\left(1-e^{-2^{n} \kappa^{n} t^{n} / n !}\right)\right] \\
& =2 \kappa\left(1-p_{i}(t)\right)\left(1-e^{-2^{n} \kappa^{n} t^{n} / n !}\right) \leq 2 \kappa\left(1-p_{i}(t)\right) \frac{2^{n} \kappa^{n} t^{n}}{n !}
\end{aligned}
$$

which gives

$$
p_{i}(t) \leq \frac{2^{n+1} \kappa^{n+1} t^{n+1}}{(n+1) !}-\int_{0}^{t} \frac{2^{n+1} \kappa^{n+1} s^{n}}{n !} p_{i}(s) d s
$$

for all $t \in\left[0,+\infty\left[\right.\right.$. Finally, Gronwall lemma [4, 10.5.1.3] yields $p_{i}(t) \leq$ $1-e^{-2^{n+1} \kappa^{n+1} t^{n+1} /(n+1) !}$ for all $t \in[0,+\infty[$, which proves the result.

Proof of the Theorem. Since, for every $n \in \mathbb{N}$, the cardinality of the set $\Omega_{n} \backslash \Omega_{n-1}$ is $4 n$, the preceding lemma shows that

$$
\mu(t) \leq 1+\sum_{n=1}^{\infty} 4 n\left(1-e^{-\tau^{n} t^{n} / n !}\right)
$$

for all $t \in[0,+\infty[$, where we write $\tau=2 \kappa$. 
Let $\rho>2$ and fix $\rho>\eta>2$. For every $n \in \mathbb{N}$ with $n>\eta$ let $\varphi_{n}$ and $\vartheta_{n}$ be the functions from $] 0,+\infty[$ into $\mathbb{R}$ defined by

$$
\begin{aligned}
\varphi_{n}(t) & =\frac{1-e^{-\tau^{n} t^{n} / n !}}{t^{\eta}} \\
\vartheta_{n}(t) & =\frac{\tau^{n} t^{n}}{(n-1) !} e^{-\tau^{n} t^{n} / n !}+\eta e^{-\tau^{n} t^{n} / n !}-\eta
\end{aligned}
$$

and let

$$
t_{n}=\tau^{-1}[(n-\eta)(n-1) !]^{1 / n} .
$$

It is a simple matter to show that $\vartheta_{n}$ is strictly increasing on $] 0, t_{n}$ ] and therefore

$$
0=\lim _{t \rightarrow 0} \vartheta_{n}(t)<\vartheta_{n}(t)
$$

for all $\left.t \in] 0, t_{n}\right] . \vartheta_{n}$ is easily checked to be strictly decreasing on $\left[t_{n},+\infty[\right.$ and obviously $\lim _{t \rightarrow+\infty} \vartheta_{n}(t)=-\eta$. Hence $\vartheta_{n}\left(s_{n}\right)=0$ for some $\left.s_{n} \in\right] t_{n},+\infty[$. Since $\left.\varphi_{n}^{\prime}(t)=t^{-\eta-1} \vartheta_{n}(t) \forall t \in\right] 0,+\infty\left[\right.$, it may be concluded that $\varphi_{n}$ is strictly increasing on $\left.] 0, s_{n}\right]$ and it is strictly decreasing on $\left[s_{n},+\infty[\right.$. Consequently, we have

$$
\begin{aligned}
& \left.\left.0=\lim _{t \rightarrow 0} \varphi_{n}(t)<\varphi_{n}(t) \leq \varphi_{n}\left(s_{n}\right) \quad \forall t \in\right] 0, s_{n}\right], \\
& 0=\lim _{t \rightarrow+\infty} \varphi_{n}(t)<\varphi_{n}(t) \leq \varphi_{n}\left(s_{n}\right) \quad \forall t \in\left[s_{n},+\infty[\right.
\end{aligned}
$$

and therefore

$$
0<\varphi_{n}(t) \leq \frac{1-e^{-\tau^{n} s_{n}^{n} / n !}}{s_{n}^{\eta}} \leq \frac{1}{s_{n}^{\eta}}<\frac{1}{t_{n}^{\eta}}=\frac{\tau^{\eta}}{[(n-\eta)(n-1) !]^{\eta / n}}
$$

for all $t \in] 0,+\infty\left[\right.$ and $n>\eta$. On the other hand, $\sum_{n=1}^{\infty} n^{1-\eta}<+\infty$ and by the Stirling formula

$$
\lim _{n \rightarrow+\infty} \frac{\tau^{\eta} n^{\eta}}{[(n-\eta)(n-1) !]^{\eta / n}}=(\tau e)^{\eta} .
$$

Consequently,

$$
M=\sum_{n=1}^{\infty} 4 n \frac{\tau^{\eta}}{[(n-\eta)(n-1) !]^{\eta / n}}=\sum_{n=1}^{\infty} 4 n^{1-\eta} \frac{\tau^{\eta} n^{\eta}}{[(n-\eta)(n-1) !]^{\eta / n}}<+\infty .
$$

Set $m \in \mathbb{N}$ with $m>\eta$. From (1) it may be concluded that

$$
\begin{aligned}
\frac{\mu(t)}{t^{\rho}} & \leq \frac{1+4(1+\cdots+m)}{t^{\rho}}+\frac{1}{t^{\rho-\eta}} \sum_{n=m+1}^{\infty} 4 n \varphi_{n}(t) \\
& \leq \frac{1+4(1+\cdots+m)}{t^{\rho}}+\frac{M}{t^{\rho-\eta}}
\end{aligned}
$$

for all $t \in] 0,+\infty\left[\right.$ and therefore $\lim _{t \rightarrow+\infty} \frac{\mu(t)}{t^{\rho}}=0$. 


\section{References}

[1] Bramson, M. and Griffeath, D., The assymptotic behavior of a probabilistic model for tumor growth: In "Biological Growth and Spread", Lecture Notes in Biomath. 38 (1979), Springer-Verlag, Berlin, 165-172.

[2] Bramson, M. and Griffeath, D., On the Williams-Bjerknes tumour growth model: II, Math. Proc. Cambridge Philos. Soc. 88 (1980), 339-357.

[3] Bramson, M. and Griffeath, D., On the Williams-Bjerknes tumour growth model: I, Ann. Probab. 9 (1981), 173-185.

[4] Dieudonné, J., Foundations of Modern Analysis, Academic Press, New York, 1960.

[5] Mollison, D., Spatial contact models for ecological and epidemic spread, J. Roy. Statist. Soc. Ser. B 39 (1977), 283-326.

[6] Richardson, D., Random growth in a tessellation, Math. Proc. Cambridge Philos. Soc. 74 (1973), 515-528.

[7] Schwartz, D., Applications of duality to a class of Markov processes, Ann. Probab. 5 (1977), 522-532.

[8] Williams, T. and Bjerknes, R., Stochastic model for abnormal clone spread through epithelial basal layer, Natur. J. 236 (1972), 19-21.

F. MARTINEZ

Departamento de Estadistica

E I.O.

Facultad DE Ciencias

UNIVERSIDAD DE GRANADA

18071 Granada, SPAIN

E-MAIL:FALVAREZ@GOLIAT.UGR.ES
A.R. Villena

Departamento DE ANALISIS

Matematico

Facultad De Ciencias

UNIVERSIDAD DE GRANADA

18071 Granada, SPAIN

E-MAIL: AVILLENA@GOLIAT.UGR.ES 